\title{
Cord blood as natural donor source for curing HIV and Leukemia
}

\author{
Jürgen Kuball \\ University Medical Center, Utrecht, Netherlands
}

Professor J. Kuball, Chairman, Department of Hematology, University Medical Center Utrecht, Room number Q05.4.301, PO Box 85500, 3508 GA UTRECHT, The Netherlands.

Phone: +31 (0) 88-75-59-030

E-mail:

In February 2007, Hütter et al performed an allogeneic hematopoietic stem cell transplantation (HSCT) in a patient with acute myeloid leukemia (AML) who has been co-infected with HIV. The HSCT was performed using peripheral blood stem cells from an adult donor. The patient, known as "The Berlin Patient", was cured of HIV as well as his AML [1]. This "magic" cure inspired many hematologists to more thoroughly investigate the prospects of cure for patients suffering from leukemia and co-infection with of HIV.

The rationale for such treatment is based on the assumption that (A) HIV primarily infects the hematopoetic system; and (B) that an HSCT replaces the infected hematopoiesis with an un-infected bone marrow. Uninfected littermates of the new bone marrow will then again repopulate the host in all different compartments of the body. However, a major challenge remains the reinfection of donor cells after HSCT. In particular typical reservoirs of HIV like monocytes are usually replaced not until months after HSCT through the new donor. Persisting host monocytes are consequently a source for HIV and threat for reinfection of the new donor. A graftversus-host disease (GVHD) may therefore be an essential factor for eliminating residual HIV reservoirs after HSCT.

To prevent HIV reinfection of the new donor in the patients, one may use a donor with a natural deletion in CCR5, which blocks entry for the most common HIV strain. This genetic trait is found in $<1 \%$ of general population. Therefore, a search for patient two has been challenging. In this case, the patient needs to have an indication for an HSCT, to be co-infected with HIV, and HIV must selectively be restricted to the receptor CCR5. The frequency of CCR5-del32/del32 donors is usually $\leq 1 \%$ in Caucasians and much less in other ethnic groups. Hence, the transplant patients usually have only a small number of available donors. When adult donors are used, a very close HLA

\section{Keywords}

HIV, leukemia, stem cell transplantation, cord blood cells.

match with the patient is required (7-8/8). Due to these limitations, the second patient was acquired only many years later in 2014 [3,8], unfortunately, with subsequent failure in terms of HIV control, most likely due to the fact that the virus already used another entry port like CXCR4.

Utilizing cord blood donors in the last decade as standard donor for many transplantations opened an avenue to a new potential source of donors for HIV infected patients. Firstly, although not well understood, a lower HLA matching between cord blood units and patients is sufficient for a good outcome of HSCT [9]. These less restricted matching criteria increase substantially the chance to find a donor in the very same genetic pool. Vice versa, if a cord blood unit with a unique trait like CCR5-del32/del32 is identified, it can be theoretically used for more patients. Increasing the pool of an inventory of CCR5-del32/del32 umbilical cord blood units will therefore provide as off-the-shelf product a rapid and greatly improved probability of finding an appropriately HLA-matched donor with an adequate cell dose for a patient with HIV infection with CCR 5 tropism and in need of an HSCT. A theoretic inventory for a minimal required number del32/del32 CB units was performed recently [7] showing that it is very likely to identify one HLA matched homozygous CCR5-del32 cord blood unit for a patient with appropriate clinical indications for HSCT. However, stem cells per cord blood unit are small and one unit is frequently not sufficient to appropriately engraft an adult patient in time. Therefore, cord blood transplantations in adults utilize usually two cord blood units in order to overcome the limitation of little numbers in stem cells per cord blood unit. As simplistic and smart this strategy seems to be at a first sight for patients without co-infection of HIV, as problematic it is for patients with HIV: It is very unlikely to find two CCR5-del32 cord blood units and outgrowth of the CCR5-del32 unit would be then left to chance. 
One solution might come from combining cord blood and haplo transplantation. The haplo product, once deeply $\mathrm{T}$ cell depleted can be considered in this strategy as a bridge to long term engraftment of the cord blood unit as pioneered by Spanish colleagues [6]. Therapeutic prospects of the combined haplo cord blood transplantation include a safer and more rapid engraftment of neutrophils, thus providing better protection against infections. Combining haplo and cord blood donors may be justified even with a suitable donor for defined indications. Others showed that the combined haplo and cord blood transplantation have an acceptable safety profile which is comparable to MUD donors $[4,5]$. However, the most recent development of single cord blood expansion protocols might provide a new avenue to pursue such transplantations with only one CCR5-del32 cord blood unit.

Beside a careful evaluation of donor choices an additional important consideration arises from the perspective of HIV positive patients: Although HIV infection is nowadays regarded as a chronic disease in the era of effective HAART, quality of life of HIV-infected patients is usually substantially impaired. A study examining the frequency of suicides in HIV positive patients from 1988 to 2008 followed 15,275 HIV-infected patients up for a median duration of 4.7 years. Suicide rates in HIV-infected individuals were more than 3 times higher than the general population [2], indeed reflecting unfortunately many restrictions experienced by HIV infected individuals during daily life.

Guiding a transplantation procedure if no state of the art is available might benefit from utilizing JACIE guidelines, a system implemented to ensure quality and improve state-ofthe-art transplantations. I.e., introduction of additional quality procedures such as an "alternative transplantation procedure" with a separate informed consent and quality controls are included in our JACIE-accredited transplantation program at the UMC Utrecht. A standard informed consent for biobanking (ethical approval) is applied for all allogeneic stem cell transplantation procedures and facilitates maximizing the acquired information regardless of the outcome of the transplantation. Multiple patients have now been transplanted with a combined haplo and cord blood protocol in Europe, while one patient received a CCR del32/32 unit at our center. The outcome of this transplantation will be reported separately.

\section{Conclusions}

CCR5-del32/del32 donors are clearly the preferential option for patients with hematological malignancies and HIV co-infection. Extending the pool of CCR5-del32/del32 cord blood units is urgently needed. Combined haplo- and cord blood transplantation or single cord blood expansion protocols can become platforms for patients with hematological malignancies and HIV co-infection. However, HSCT puts the patient at a high risk, thus cannot be considered as solution for a functional cure of HIV. Beside curing a patient of HIV, this type of transplantation allows studying HIV repertoires which is important to develop mechanisms of cure for HIV.

Our and other cases started a very interesting international collaboration; the "epistem-project": http://www.epis- tem-project.org, funded by AMFAR, a European Project to guide the therapy and investigate the potential for HIV cure by allogeneic stem cell transplantation. Its areas of investigations are as follows: (1) Increasing the number of suitable del32/del32 CB units by testing systematically European cord blood banks; (2) Providing advice during treatment (donor choice, ethical aspects, drug-drug interaction, etc.); (3) Improving the understanding of biology for HIV cure by providing a standardized sampling protocol which will allow comparable studies of viral reservoirs, characterization of infecting virus, and evaluation of immune responses after allo SCT between different transplantation centers.

\section{References}

1. Hutter G, Nowak D, Mossner M, Ganepola S, Mussig A, Allers K, Schneider T, Hofmann J, Kucherer C, Blau O. Longterm control of HIV by CCR5 Delta32/Delta32 stem-cell transplantation. N Engl J Med 2009; 360: 692-8.

2. Keiser O, Spoerri A, Brinkhof MW, Hasse B, Gayet-Ageron A, Tissot F, Christen A, Battegay M, Schmid P, Bernasconi E et al. Suicide in HIV-infected individuals and the general population in Switzerland, 1988-2008. Am J Psychiat 2010; 167: 143-50.

3. Kordelas L, Verheyen J, Beelen DW, Horn PA, Heinold A, Kaiser R, Trenschel R, Schadendorf D, Dittmer U, Esser S et al. Shift of HIV tropism in stem-cell transplantation with CCR5 Delta32 mutation. New Engl J Med 2014; 371: 880-2.

4. Kwon M, Balsalobre P, Serrano D, Perez CA, Buno I, Anguita J, Gayoso J, ez-Martin JL. Single Cord Blood Combined with HLA-Mismatched Third Party Donor Cells: Comparable Results to Matched Unrelated Donor Transplantation in High-Risk Patients with Hematologic Disorders. Biol Blood Marrow Transplant 2013; 19(1): 143-9.

5. Liu H, Rich ES, Godley L, Odenike O, Joseph L, Marino S, Kline J, Nguyen V, Cunningham, J, Larson RA et al. Reduced-intensity conditioning with combined haploidentical and cord blood transplantation results in rapid engraftment, low GVHD, and durable remissions. Blood 2011; 118: 6438-45.

6. Magro E, Regidor C, Cabrera R, Sanjuán I, Forès R, Garcia-Marco JA, Ruiz E, Gil S, Bautista G, Millán I, Madrigal A, Fernandez MN. Early hematopoietic recovery after single unit unrelated cord blood transplantation in adults supported by co-infusion of mobilized stem cells from a third party donor. Haematologica 2006; 91(5): 640-8.

7. Petz LD, Redei I, Bryson Y, Regan D, Kurtzberg J, Shpall E, Gutman J, Querol S, Clark P, Tonai R et al. Hematopoietic cell transplantation with cord blood for cure of HIV infections. Biol Blood Marrow Transplant 2013; 19: 393-7.

8. Verheyen J, Esser S, Kordelas L. More on shift of HIV tropism in stem-cell transplantation with CCR5 delta32/delta32 mutation. New Engl J Med 2014; 371: 2438.

9. Wagner JE, Jr, Eapen M, Carter S, Wang Y, Schultz KR, Wal, DA, Bunin N, Delaney C, Haut P, Margolis D et al. Oneunit versus two-unit cord-blood transplantation for hematologic cancers. New Engl J Med 2014; 371: 1685-94. 